\title{
The USP18 cysteine protease promotes HBV production independent of its protease activity
}

\author{
Yujia $\mathrm{Li}^{1 \dagger}$, Min $\mathrm{YaO}^{2 \dagger}$, Xiaoqiong Duan ${ }^{1}$, Haiyan Ye ${ }^{1}$, Shilin $\mathrm{Li}^{1}$, Limin Chen ${ }^{1,3}$, Chunhui Yang ${ }^{1 *}$ and Yongjun Chen ${ }^{1 *}$
}

\begin{abstract}
Background: Hepatitis B virus (HBV) infection remains as one of the major public health problems in the world. Type I interferon (IFN) plays an essential role in antiviral defense by induced expression of a few hundred interferon stimulated genes (ISGS), including ubiquitin-specific protease 18 (USP18). The expression level of USP18 was elevated in the pretreatment liver tissues of chronic hepatitis $\mathrm{B}(\mathrm{CHB})$ patients who did not respond to IFN treatment. Thus, this study was designed to investigate the effects of USP18 on HBV replication/production.

Methods: The levels of wild type USP18(WT-USP18) and USP18 catalytically inactive form C64S were up-regulated by plasmids transfection in HepAD38 cells, respectively. Real-time PCR and ELISA were used to quantify HBV replication. Type I IFN signaling pathway was monitored at three levels: p-STAT1 (western Blot), interferon stimulated response element (ISRE) activity (dual luciferase assay) and ISGs expression (real time PCR).

Results: Our data demonstrated that overexpression of either WT-USP18 or USP18-C64S inactive mutant increased the intracellular viral pgRNA, total DNA, cccDNA, as well as HBV DNA levels in the culture supernatant, while silencing USP18 led to opposite effect on HBV production. In addition, upregulated WT-USP18 or USP18-C64S suppressed ISRE activity and the expression levels of p-STAT1 and ISGs.
\end{abstract}

Conclusion: USP18 promoted HBV replication via inhibiting type I IFN signaling pathway, which was independent of its protease activity.

Keywords: HBV, Interferon, USP18, Type I IFN signaling pathway, Persistent infection

\section{Background}

As one of the major public health problems, Hepatitis B virus (HBV) infected about 257 million people worldwide, leading to hepatic fibrosis, cirrhosis, and even hepatocellular carcinoma (HCC). Type I interferons (IFNs), the key effector of innate immunity, have long been used to treat different viral diseases such as $\mathrm{HBV}$ and Hepatitis $\mathrm{C}$ virus (HCV) infections. Although the functions of $\mathrm{HBV}$ proteins are increasingly

\footnotetext{
* Correspondence: yangchunhui@ibt.pumc.edu.cn;

chenyongjun@ibt.pumc.edu.cn

${ }^{\dagger}$ Yujia Li and Min Yao contributed equally to this work.

1 Institute of Blood Transfusion, Chinese Academy of Medical Sciences and

Peking Union Medical College, Chengdu 610052, Sichuan, China

Full list of author information is available at the end of the article
}

understood, the role of host factors in modulating HBV infection remains elusive.

Increasing evidence puts IFN stimulated gene 15/ ubiquitin-specific protease 18 (ISG15/USP18) system, both of which are abundantly induced by type I IFN, at the center of regulatory processes of host innate immune response (reviewed in [1]). ISG15 plays its role in antiviral defense and immunoregulation during pathogen evasion either through conjugating to host or pathogen proteins (ISGylation) [2] or function as a cytokine in free form [3]. As the major ISG15 de-conjugating enzyme, USP18 is an off-switch in ISGylation, striping ISG15 from its target proteins (known as deISGylation) and 
playing important role in viral infections through its ISG15 protease activity [4]. However, recent evidence suggested that USP18 might play its role independent of the protease activity: USP18 was able to bind to the subunit 2 of type I IFN receptor to inhibit IFN-induced Janus kinase/signal transducer and activator of transcription (Jak/STAT) signaling pathway [5].

Our previous data $[6,7]$ showed that chronic HCV patients, who had high pretreatment hepatic expression of a subset of interferon stimulated genes (ISGs), including ISG15 and USP18, did not respond to standard pegylated IFN $\alpha /$ Ribavirin (PegIFN/Rib) treatment. Similarly, our clinical data revealed that chronic hepatitis B $(\mathrm{CHB})$ patients who had pre-treated higher hepatic USP18 expression responded poorly (or called "non-response, NR") to IFN treatment [8]. Several groups also investigated the effects of USP18 suppression on HBV replication. Using in vivo HBV replication mouse model, Kim $\mathrm{JH}$ et al. [9] demonstrated that blocking ISGylation by UBE1L silencing exhibited very little effect on HBV replication, while USP18 knockout significantly suppressed HBV production. One more recent study [10] reported that silencing USP18 in HepG2.2.15 cells decreased the intracellular HBV pgRNA level and inhibited secretion of HBV DNA, HBsAg and HBeAg into the supernatant. However, very little study focused on the influence of USP18 overexpression on HBV replication/production. In the present study, we explored whether increased USP18 expression affected HBV replication in HepAD38 cells which, to some extent, resembled the persistent viral replication situation of the NR patients with high expression of USP18 in liver.

\section{Materials and methods \\ Cell lines}

HepG2 cells and Hela cells were cultured in Dulbecco's Modified Eagle's Medium (DMEM) (Gibco, USA) supplemented with $10 \%$ fetal bovine serum (FBS) (Gibco, USA), penicillin (100 IU $/ \mathrm{ml}$; Gibco, USA), and streptomycin $\left(100 \mu \mathrm{g} / \mathrm{ml}\right.$; Gibco, USA) in $5 \%$ CO2 incubator at $37^{\circ} \mathrm{C}$. HepAD38 cells, derived from HepG2 cells by integrating full-length HBV genome in the cellular genome, support HBV expression under the control of a tetracyclineregulated (tet-off) promoter [11], were kindly provided by Professor Bo Qin and Dr. Zeng Tu (Chongqing Medical University, China). The cells were routinely cultured as mentioned above. Additionally, HepAD38 cells were treated with G418 for resistance screening.

\section{Plasmids construction}

Human full-length USP18 gene fused to GFP at the Nterminus was cloned into pcDNA-DEST53 (Invitrogen, USA). Briefly, human USP18 ORF (in pENTER221 entry vector, Invitrogen, USA) was cloned into the destination vector (PcDNA-DEST53) by LR recombination (wide type USP18, WT-USP18). Western blot was used to confirm USP18 protein expression (anti-USP18 antibody). The USP18 mutant form C64S (USP18-C64S) was obtained by in vitro mutagenesis using the GeneTailor kit (Invitroge, USA). The primers used were: USP18 forward for C64S 5'-caacattggacagaccagctgccttaactccttga- 3 ' and USP18 reverse for C64S 5 '-ggtctgtccaatgttgtgtaaaccaaccaggccat-3'. After methylation, pENTER221-USP18 was used as a template for PCR reactions using the above mutant primer pairs, and the resulting mutant form was screened on LB/Agar plates containing $100 \mu \mathrm{g} / \mathrm{ml} \mathrm{kana-}$ mycin. All constructs generated in this study were confirmed by DNA sequencing across joints.

\section{Confirmation of ISG15-cleavage activity of USP18 in vitro} ISG15/glutathione S-transferase (GST) expression plasmid was created by cloning the ISG15/GST fusion gene into pcDNA4/HisMax vector (Invitrogen, USA). Real-time PCRs was performed by employing faststart Universal SYBR Green Master Mix (Roche, USA) following the manufacturer's protocols to determine expressions of ISG15 which was further confirmed by Western blot [12]. ISG15cleavage activity of wild type USP18 (WT-USP18) and USP18-C64S were analyzed by co-transfection of ISG15/ GST and WT-USP18 (or USP18-C64S) into Hela cells.

\section{Enyzme-linked immunosorbent assay (ELISA)}

The levels of HBsAg and HBeAg in the culture supernatant of HepAD38 were detected by Human HBsAg and HBeAg ELISA Kits (Andy Gene, China) following the manufacturer's instructions. The expression levels of IFN $\alpha$ and IFN $\beta$ in culture supernatant of HepG2 cells and HepAD38 cells were measured by Human IFNa/ IFN $\beta$ ELISA Kits (Shanghai jijin Chemistry Technology, China),respectively.

\section{USP18 knockdown}

USP18 small inhibitory RNA (siUSP18: 5'-CUGCAU AUCUUCUGGUUUATT-3') and the negative control (NC) siRNA (NC: 5'-UUCUCCGAACGUGUCACGUTT-3') were purchased from Sangon Biotech, China. HepAD38 cells were seeded at $3 \times 10^{5} / \mathrm{ml}, 2 \mathrm{ml}$ per well in 6-well plates. $24 \mathrm{~h}$ later, the cells were left untreated or transfected with $20 \mathrm{nM}$ siUSP18 or $20 \mathrm{nM} \mathrm{NC}$ by using Lipofectamine ${ }^{\circ}$ RNAiMAX (Invitrogen, USA) reagent according to the manufacturer's instructions. $48 \mathrm{~h}$ post transfection, intracellular total protein was extracted to detect USP18 expression by western blot. Intracellular total RNA and DNA, as well as supernatant DNA, were collected respectively. Real-time PCR was performed to detect HBV pgRNA, USP18 mRNA, cccDNA and intracellular/supernatant HBV total DNA. 
Table 1 Real-Time PCR Primes

\begin{tabular}{ll}
\hline Gene Name & Nucleotide sequence \\
\hline GAPDH & $\begin{array}{l}\text { Forward 5'-GCCTCCTGCACCACCAACTG-3' } \\
\text { Reverse 5'-ACGCCTGCTTCACCACCTTC-3' }\end{array}$ \\
USP18 & Forward 5'-CAGACCCTGACAATCCACCT-3' \\
& Reverse 5'-AGCTCATACTGCCCTCCAGA-3' \\
HBV total DNA & Forward 5'-CGTTITGCCTTCTGACTTCTTC-3' \\
& Reverse 5'-ATAGGATAGGGGCATTGGTGGTC-3' \\
HBV ccCDNA & Forward 5'-TTCTCATCTGCCGGACCG-3' \\
HBV pgRNA & Reverse 5'-CACAGCTTGGAGGCTTGAAC-3' \\
& Forward 5'-CTCAATCTCGGGAATCTCAATGT-3' \\
MxA & Reverse 5'-TGGATAAAACCTAGCAGGCATAAT-3' \\
& Forward 5'-GTGCATTGCAGAAGGTCAGA-3' \\
OAS2 & Reverse 5'-CTGGTGATAGGCCATCAGGT-3' \\
& Forward 5'-TCAGCGAGGCCAGTAATCTT-3' \\
& Reverse 5'-GCAGGACATTCCAAGATGGT-3' \\
\hline
\end{tabular}

USP18 Ubiquitin specific protease $18, H B V$ ccCDNA hepatitis B virus covalently closed circular DNA, pgRNA hepatitis B virus pregenomic RNA, MxA myxovirus resistance protein A, OAS2 2',5'-oligoadenylate synthetase 2

\section{RNA/DNA isolation and quantitative real-time polymerase} chain reaction

Total RNA was extracted from HepAD38 cells using Trizol (Invitrogen, USA) and the cDNAs were synthesized by reverse transcription using ReverTraAce qPCR
RT Master Mix (TOYOBO, Japan) following the manufacture's protocol. Quantitative real-time PCR was performed using the SYBR Green Real-time PCR Master Mix (TOYOBO, Japan). Total intracellular DNA in cells were acquired by TIANamp Genomic DNA Kit (TIANGEN, China) while supernatant viral DNA were extracted by QIAamp DNA Blood Mini Kit (Qiagen, German). The forward and reverse primers specific for the genes are shown in Table 1. HBV DNA, pgRNA, and some ISGs were quantified by real-time PCR according to the instructions.

\section{Western blot analysis}

Western blot was performed to detect the semiquantification of ISG15、USP18 and HBV protein levels. The primary antibodies used were as follows, rabbit anti-ISG15 (CST, USA), mouse anti-GAPDH (Zheng De, China), mouse anti- $\beta$-actin (CST, USA), mouse antiHBcAg (Boster Biological Technology, China), rabbit anti-p-STAT1 (Tyr701) (CST, USA) and rabbit antiSTAT1 (CST, USA). For USP18, two kinds of primary antibodies were used: USP18 Polyclonal Antibody (Invitrogen, USA) (one band) and rabbit anti-USP18 (CST, USA) (two bands). Secondary antibodies were HRP-
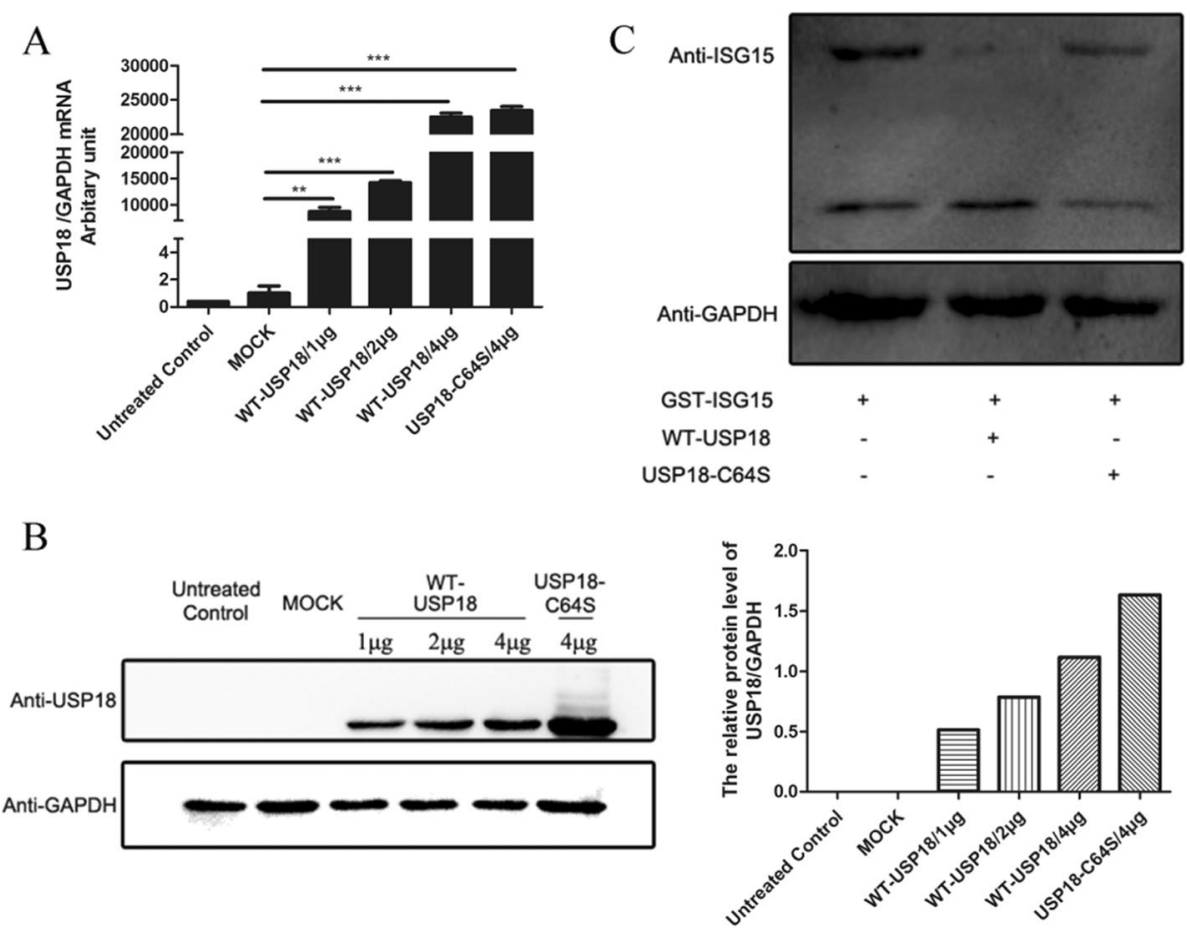

Fig. 1 Over-expression of USP18 and its catalytic activity. HepAD38 cells were transfected with WT-USP18 plasmid, USP18-C64S plasmid or empty vector (MOCK) or left untreated. a: Twenty-four hours after transfection, USP18 mRNA was determined by real-time PCR (normalized by GAPDH). b: Forty-eight hours after transfection, USP18 protein expressions were analyzed by western blot (left). The relative expression levels of USP18 (normalized by GAPDH) were calculated by densitometry analysis (right). c: Cleavage of ISG15-GST fusion in vitro. USP18, ISG15/GST and WTUSP18 (or USP18-C64S) were co-transfected into Hela cells. Total intracellular protein was collected to perform Western blot. WT-USP18, wide type USP18; MOCK, empty plasmid. Results are presented as means \pm SD $(n \geq 3) .{ }^{* *} p \leq 0.01 ;{ }^{* * *} p \leq 0.001$ 
labeled goat anti-mouse (Biosharp, China) or anti-rabbit IgG (Beyotime, China). The protein bands were visualized using an ECL chemiluminescent detection kit (Millipore, USA) by ChemiDocTM Imaging Systerm (BIO-RAD, USA). The relative intensities of protein bands were analyzed with Image $2 \times 2.1 .4 .7$ software.

\section{Dual-luciferase report gene system}

HepAD38 cells were seeded at a density of $3.0 \times 10^{5}$ per well in 24-well plates. Twenty-four hours later, $0.5 \mu \mathrm{g}$ ISRE (interferon stimulated response element)-luc reporter plasmid and $2 \mathrm{ng}$ PRL-TK reporter plasmid were co-transfected with $1 \mu \mathrm{g}$ pcDNA3.1-3*tag plasmid (MOCK) or $1 \mu \mathrm{g}$ USP18 plasmid. Twelve hours after transfection, the culture medium was removed and replenished with fresh medium. Twenty-four hours post transfection, cells were treated with IFN $\alpha(0 \mathrm{IU} / \mathrm{ml}, 100$ $\mathrm{IU} / \mathrm{ml}$ and $1000 \mathrm{IU} / \mathrm{ml}$ ) for additional $24 \mathrm{~h}$. Then, cells were lysed with passive lysis buffer and the relative luciferase activity was detected by Dual-Luciferase Reporter(DLR) Assay kit (Promega, USA) according to the manufacturer's protocol.

\section{Statistical analysis}

All experiments in this study were performed at least three independent times. Statistical differences were compared by Student's t-test through GraphPad Prism software. $p$ values $\leq 0.05$ were considered statistically significant.

\section{Results}

\section{Confirmation of USP18 expression and its catalytic} activity

In order to explore the effect of USP18 on HBV infection, we first confirmed whether USP18 and USP18-C64Secoding plasmids were successfully constructed. Figure 1a showed that transfection of WT-USP18 or USP18-C64S plasmid led to a pronounced increase of USP18 mRNA expression in a dose-dependent manner, which was further confirmed by western blot (Fig. 1b). The transfection efficiency was shown by the GFP expression in the cells (Supplement Fig. 1). Since it has been reported [13] that full length USP18 has a conserved catalytic-activity-related site cysteine at Cys64 in its Cys-box, we obtained the mutant form of USP18 by conversing the cyserine into serine. And then the Hela cells, in which ISGylation could not be induced because of lacking E1 activating enzyme Ube1L

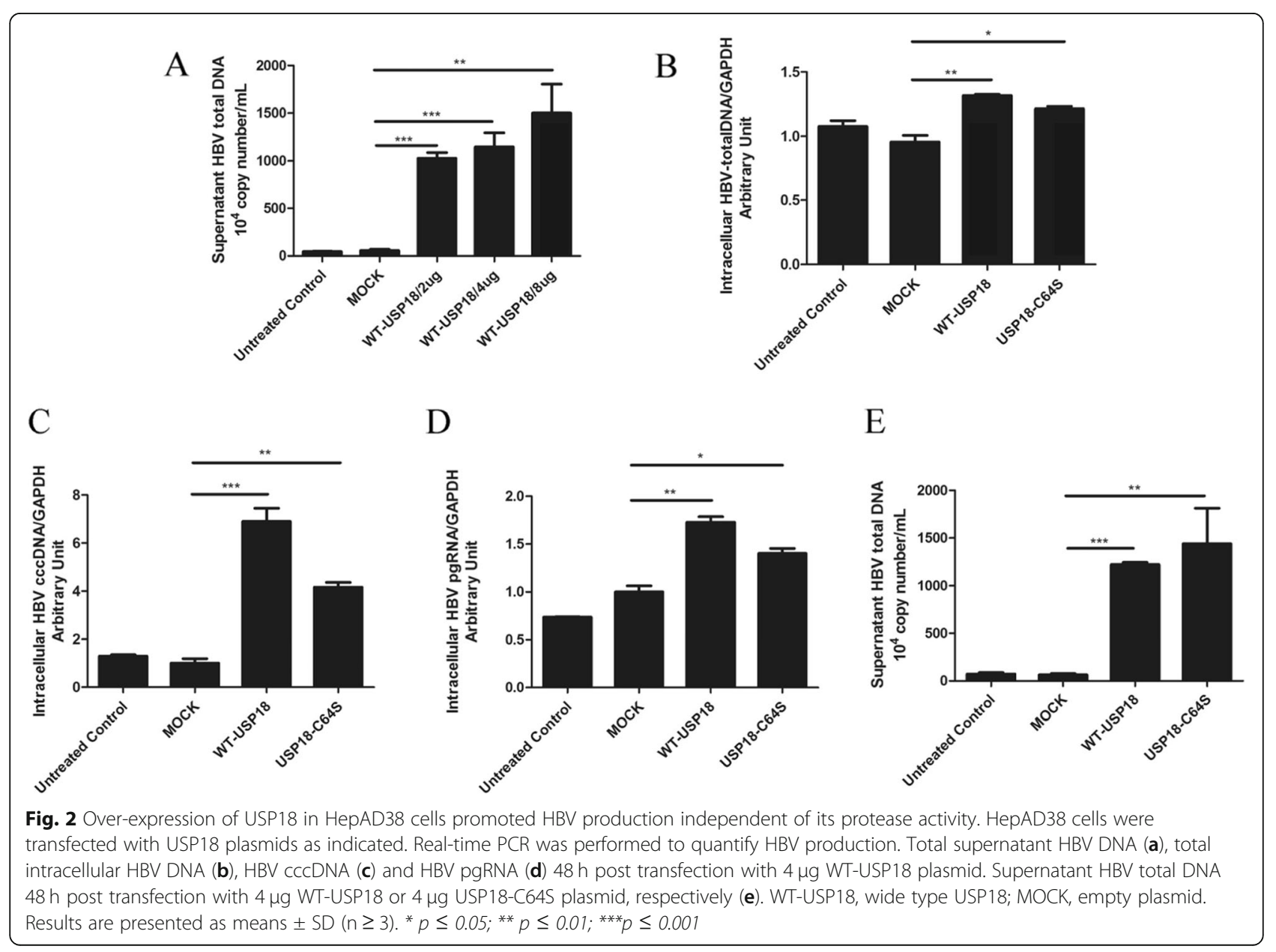


[14], were co-transfected with the pcDNA4/HisMaxISG15/GST and WT-USP18 or USP18-C64S plasmids. Western blot showed two bands of ISG15: the upper GST-ISG15 band and the lower ISG15 band, which indicated the expression of WT-USP18 led to release of the ISG15 protein from its conjugated GST-ISG15, while USP18-C64S did not (Fig. 1c).

\section{USP18 regulated HBV production independent of its protease activity}

To evaluate the effect of USP18 on HBV replication, we analyzed the expression levels of supernatant $\mathrm{HBV}$ DNA, intracellular HBV pgRNA, total HBV DNA and cccDNA, following USP18 over-expression in HepAD38 cells. The results showed that total supernatant HBV DNA was dramatically increased in parallel with USP18 up-regulation in a dose-dependent manner (Fig. 2a). And the expression of intracellular HBV DNA (Fig. 2b), cccDNA (Fig. 2c), as well as the pgRNA (Fig. 2d) were also elevated with ectopic USP18 expression. We then asked whether the catalytic ability of USP18 was responsible for enhanced HBV replication. As expected, either WT-USP18 or USP18-C64S catalytically inactive mutant could stimulate HBV production (Fig. 2e), indicating that USP18 stimulated HBV production independent of its proteolytic capacity. More interestingly, USP18 showed very limited effect on HBsAg (Fig. 3a), HBeAg (Fig. 3b) in the supernatant and Intracellular HBcAg (Fig. 3c). We also evaluated the effect of USP18 silencing on HBV production. USP18 mRNA expression was significantly suppressed by RNA interference (Fig. 4a, left), which was further confirmed by western blot (Fig. 4a, right). And as expected from the experimental design, the supernatant (Fig. 4b) and Intracellular (Fig. 4d) total HBV DNA, cccDNA (Fig. 4e), as well as pgRNA (Fig. 4f) levels were decreased significantly in parallel with USP18 silencing, while the $\mathrm{HBsAg}, \mathrm{HBeAg}$ and intracellular HBcAg remained unaffected (Supplement Fig. 2).

Over expression of USP18 inhibited IFN-induced Jak/STAT signaling pathway

Due to the fact that USP18 is a negative modulator of type I IFN signaling pathway, we hypothesize that endogenous type I IFN has been induced by HBV embraced in HepAD38 cells to defense viral replication and over-expression of USP18 may suppress the activation of Jak/STAT signaling pathway, consequently facilitating HBV production. In order to prove this hypothesis, we firstly measured the expression of type I IFN in HepAD38 and HepG2 cell culture medium by ELISA. As shown in Fig. 5a, although no difference was found in IFN $\beta$ expressions between these two cell lines, statistically significant potentiation of IFN $\alpha$ expression was found in HepAD38 cells compared with that in HepG2 cells. Real-time PCR further revealed that the expression of typical ISGs (MxA and OAS2) was increased in HepAD38 cells compared to HepG2 cells (Fig. 5b),
A

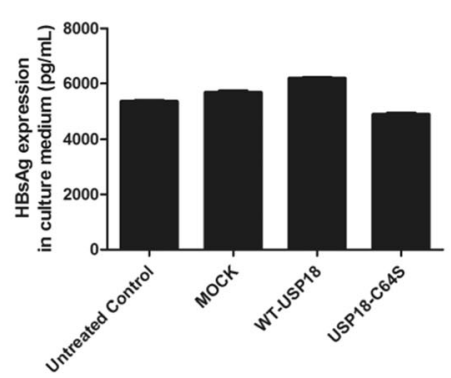

B

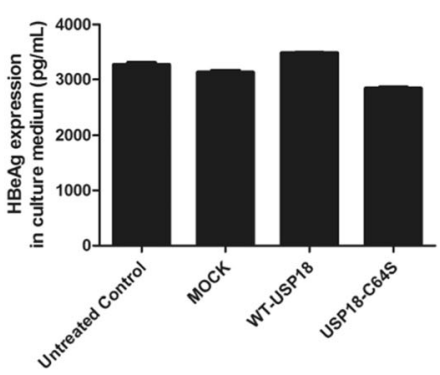

$\mathrm{C}$
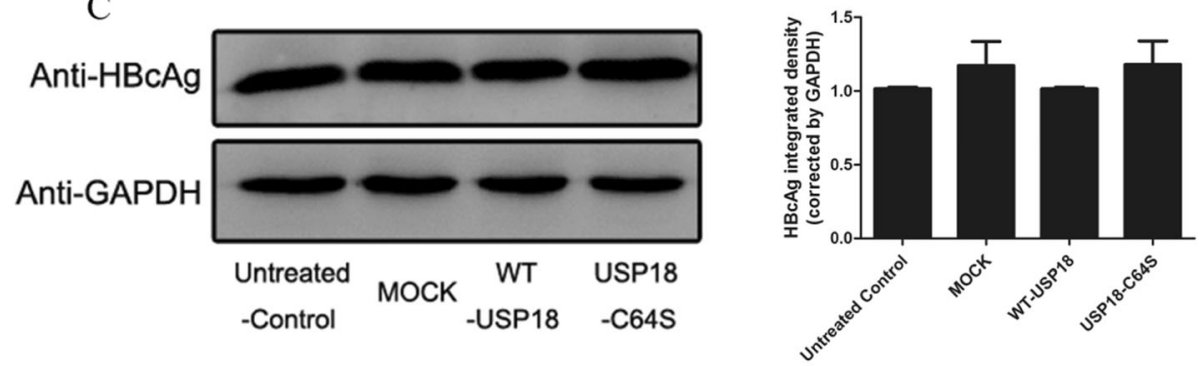

Fig. 3 Over-expression of USP18 in HepAD38 cells did not affect expression of HBV proteins. HepAD38 cells were transfected with the $4 \mu \mathrm{g}$ WTUSP18, $4 \mu \mathrm{g}$ USP18-C64S or $4 \mu \mathrm{g}$ MOCK, respectively. Forty-eight hours later, culture medium was collected to quantify HBsAg (a) and HBeAg (b) expression level by ELISA assay. Intracellular HBCAgwas detected by western blot (c, left) and (c, right) analyzed by densitometry analysis. WTUSP18, wide type USP18; MOCK, empty plasmid. Results are presented as means \pm SD $(n \geq 3)$ 

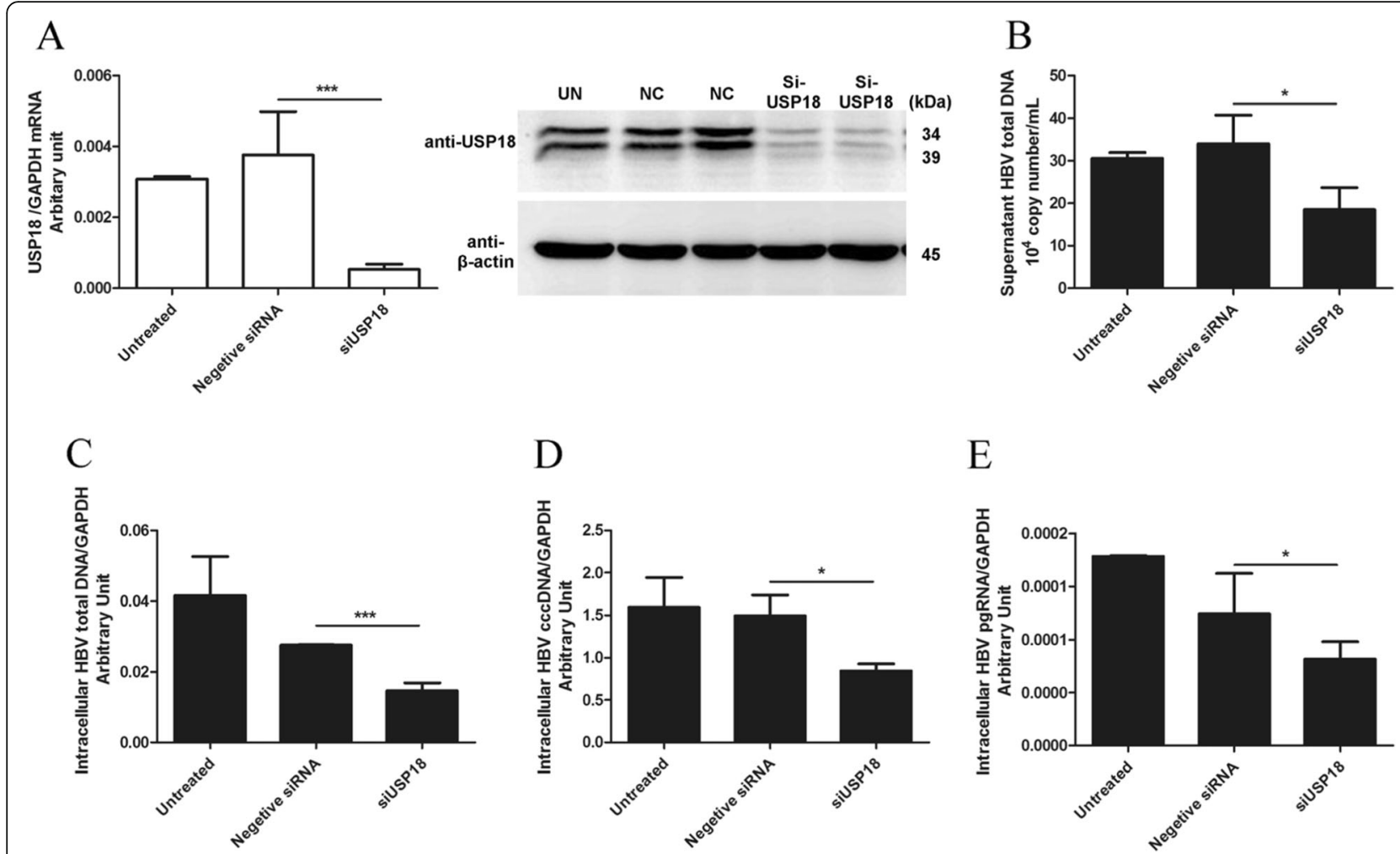

Fig. 4 Silencing USP18 in HepAD38 suppressed HBV production. HepAD38 cells were seeded at $3 \times 10^{5} / \mathrm{ml}, 2 \mathrm{ml}$ per well in 6 -well plates. $24 \mathrm{~h}$ later, the cells were left untreated or transfected with $20 \mathrm{nM}$ siUSP18 or $20 \mathrm{nM} \mathrm{NC.} 48 \mathrm{~h}$ post transfection, knockdown efficiency was evaluated by detecting USP18 mRNA expression (a, left), which was further confirmed by western blot (a, right). Intracellular total RNA and DNA, as well as supernatant DNA, were collected respectively. Real-time PCR was performed to detect supernatant HBV total DNA (b), intracellular HBV total DNA (c), HBV cccDNA (d) and pgRNA (e). siUSP18, USP18 small inhibitory RNA; NC, the negative control siRNA. Results are presented as means \pm SD $(\mathrm{n} \geq 3)$. ${ }^{*} p \leq 0.05 ;{ }^{* *} p \leq 0.01 ;{ }^{* * *} p \leq 0.001$

indicating the activated type I IFN signaling pathway in HepAD38 cells. In consistent with this, when the tetracycline-responsive replication of HBV was inhibited by tetracycline $(1 \mathrm{mg} / \mathrm{ml})$ treatment, the endogenous IFN $\alpha$ and ISG mRNA including MxA and OAS2 expression were also depressed (Supplemental Fig. 3). We then asked whether USP18 overexpression could affect Jak/ STAT signaling pathway in HepAD38 cells. Western blot, dual luciferase assay and real-time PCR were employed to evaluate the protein level of phosphorylated STAT1 (p-STAT1), ISRE activity and mRNA expression of typical ISGs, respectively. The results suggested that levels of p-STAT1 (Fig. 5c, left), ISRE activity (Fig. 5c, middle) and ISGs (MxA, OAS2) (Fig. 5c, right) were all inhibited by up-regulation of either WT-USP18 or USP18-C64S, indicating the impaired type I IFN signaling.

\section{Discussion}

Previous work from several laboratories examined the role of USP18 in innate defense to HBV infection. USP18 has been proved to be associated with HBV infection in mouse model. Either USP18 knockout mice or
USP18 knockdown mice generated by specific shRNA experienced restricted HBV replication, while the UbE1L -/- mouse which had suppressed ISG15 modification did not [9]. More recent evidence [10] suggested that silencing USP18 alone was enough to inhibit HBV expression in human HepG2.2.15 cells. In accordance with these studies, our study observed that over-expression of USP18 could promote HBV production in a catalysisunconcerned manner, which, to some extent, provided an explanation to the association between high pretreatment hepatic ISGs levels and persistent HBV infection [15]. Similarly, USP18-/- mice showed enhanced resistance to lymphocytic choriomeningitis virus (LCMV) or vesicular stomatitis virus (VSV) infection and reduced viral replication [16, 17]. Nevertheless, over-expression of USP18 significantly restrict viral replication during porcine respiratory and reproductive syndrome virus (PRRSV) infection which might be attributed to early activation of NF- $\mathrm{KB}[18,19]$. These diverse effects on virus infections implicate that USP18 functions via virus-specific and/or species-specific fashions which may be mediated by the interaction of USP18 and certain viral proteins. 

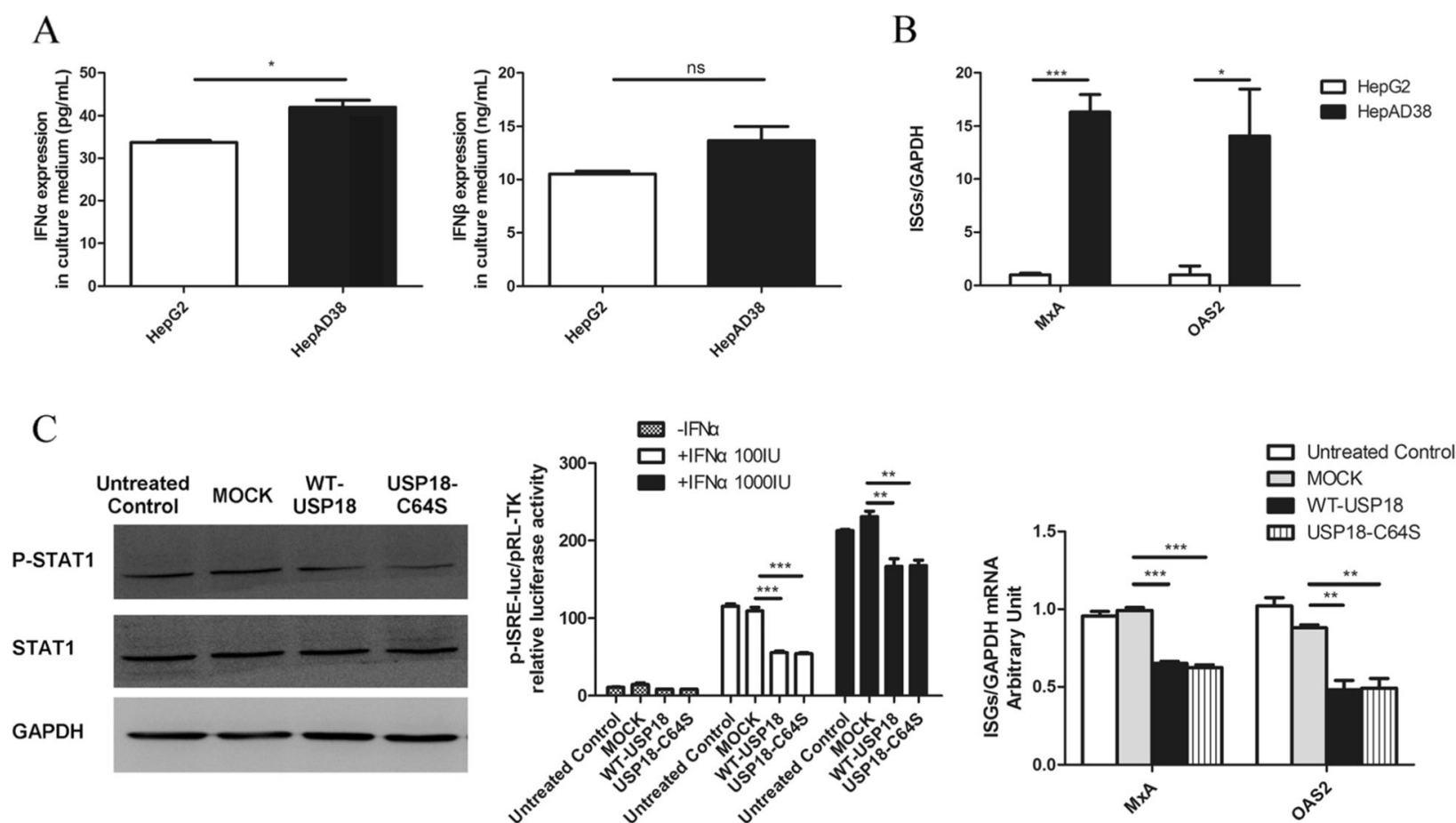

Fig. 5 USP18 upregulation inhibited IFN-induced Jak/STAT signaling pathway. HepAD38 cells and HepG2 cells were seeded in 6-well plate without any treatment, respectively. Forty-eight hours later, supernatant IFNa (a, left) and IFN $\beta$ (a, right) and intracellular mRNA expression of ISGs (b) were analyzed by ELISA assay and real-time PCR, respectively. To investigate the effects of USP18 on STAT phosphorylation, HepAD38 cells were transfected with WT-USP18, USP18-C64S or MOCK for $48 \mathrm{~h}$ and treated with $500 \mathrm{IU} / \mathrm{ml}$ IFNa for 30 min before harvested. Western blot was used to analyze the expression of STAT1 and p-STAT1 (c, left). The interferon stimulated response element (ISRE) activity was quantified by dual luciferase reporter gene assay. Briefly, HepAD38 cells were co-transfected with WT-USP18, USP18-C64S or MOCK and ISRE-IUC reporter plasmid /pRL-TK reporter plasmid for $24 \mathrm{~h}$, and then left untreated or treated with $100 \mathrm{IU} / \mathrm{ml}$ or $1000 \mathrm{IU} / \mathrm{ml} \mathrm{IFNa}$ for $24 \mathrm{~h}$ before the cells were lysed (c, middle). HepAD38 cells were transfected with WT-USP18, USP18-C64S or MOCK for $48 \mathrm{~h}$ and treated with $500 \mathrm{IU} / \mathrm{ml}$ IFNa for additional $24 \mathrm{~h}$. Expression of ISGs mRNA including MXA and OAS2 were detected by real-time PCR (c, right). WT-USP18, wide type USP18; MOCK, empty plasmid. Results are presented as means \pm SD $(n \geq 3)$. ${ }^{*} p \leq 0.05 ;{ }^{* *} p \leq 0.01 ;{ }^{* * *} p \leq 0.001$

Although responses vary between individuals [20, 21], type I IFN is still one of the most important choices in HBV therapy. Using different cell models, several groups $[5,22]$ reported that USP18 could interfere with the binding of IFN $\alpha$ to its receptor, resulting in cellular desensitization to further IFN $\alpha$ stimulation. In human cells, USP18 also have a similar role [22, 23]. Our data indicated that existence of HBV in HepAD38 cells stimulated the expression of endogenous type I IFN and subsequent signal pathway compared with that in HBV-free HepG2 cells. We speculated that this activated innate immune state, to some extent, can suppress HBV replication at a relatively low level. And over-expression of USP18 inhibited type I IFN signal pathway and broken the balance, resulting in elevated HBV replication. Furthermore, it is very interesting that overexpression of USP18 or USP18 C64S showed very little influence on intracellular $\mathrm{HBcAg}$, supernatant $\mathrm{HBsAg}$ and $\mathrm{HBeAg}$, while they promoted HBV DNA level significantly. At least two reasons might be involved: 1) The life cycle of HBV in HepAD38 cells (in vitro model) is not exactly the same as that in in vivo model or patients. With different treatment, the change of HBV nucleic acid level is not always in parallel with that of HBV protein expression levels in HepAD38 cells. It has been reported that HBV DNA in the culture medium was increased by doxycycline treatment [24] or tetherin [25] overexpression while the supernatant HBsAg level was not affected. And Kinoshita W et al. [26] found that host factor PRPF31 knockdown suppressed cccDNA production but showed little effect on HBcAg expression. 2) USP18 may affect HBV replication through different ways.

In contrast to $\mathrm{HCV}$, acute $\mathrm{HBV}$ infection seldom induced type I or type III IFN production in chimpanzees [27]. Clinical data [28] also showed that patients with acute HBV infection did not experience significant IFN expression. This phenomenon may be caused by mild replication of HBV at early stage of infection [29]. However, it may be a little bit different in the chronic infection. First of all, JAK-STAT Signaling Pathway has been demonstrated to be involved in regulating HBV replication in HepG2.2.15 cells [10, 26] and HepAD38 cells [24, 
25] with persistent HBV virion expression. Secondly, expression of IFN and upregulation of ISGs have been observed in persistent HBV infection. Dongni Gao et al. [30] found that after transfected with pHBV (containing the full-length genome of the HBV genotype C), the IFN $\alpha$ protein levels in Huh7 cells culture supernatants was up to $100 \mathrm{pg} / \mathrm{Ml}$. Our previous study also showed that the pre-treatment expression of ISGs (ISG15, MxA, etc) was significantly up-regulated in hepatocytes of some patients with chronic hepatitis B $[8,15]$. Most recently, Li L et al. [10] also reported that the expression level of USP18 in HepG2.2.15 cells was higher than that in HepG2 cells. Although more evidence is required to elucidate the potential mechanism, we could speculate that there might be a balance between the baseline activation of IFN signaling pathway and HBV replication. And once the balance is disturbed, the viral replication is promoted. Moreover, HBV DNA in the liver and blood could be cleared before onset of adaptive immune response [29], indicating that innate molecules, in addition to IFN, may contribute to controlling HBV infection. In fact, with exposure to HBV, macrophages could be activated characterizing by upregulated expression of inflammatory cytokines [31]. For example, interleukin 6 (IL-6) was reported to suppress early HBV gene expression by down-regulating the level of two transcription factors, hepatocyte nuclear factor (HNF) $1 \alpha$ and HNF $4 \alpha$ [32]. Thus, further study will be needed to investigate the effects of USP18 on these newly identified $\mathrm{HBV}$-associated innate molecules.

It is wildly accepted that USP18 could conduct functions by controlling ISGylation dependent of its protease activity. Owing to the fact that over 300 cellular proteins $[33,34]$ and increasing number of pathogen's proteins [35-38] have been identified as ISG15 targets, it is conceivable that USP18 is a key modulator involved in diverse cellular pathways and biological processes. On the other hand, however, there are multiple lines of evidence for protease-independent activities of USP18.Our findings revealed that either wide type USP18 or USP18 C64S could promote HBV production in HepAD38 cells. Klaus-Peter Knobeloch et al. [39] compared USP18-/mice with ISG15-/- and USP18-/- double-deficient mice and found that ISG15 deletion did not rescue the severe consequences, such as high mortality, neurological symptoms and hydrocephalus, resulting from USP18 deficiency. Their findings indicated that although elevated ISGylation was induced by USP18 knockout, severe phenotype of USP18-/- mice might be caused by non-ISG15 mediating mechanism rather than lack of deISGylation. Harish Potu's group [40] showed that both USP18 and its catalytically inactive form had similar anti-apoptotic effects to limit the apoptosis triggered by IFN $\alpha$ or bortezomib.

\section{Conclusions}

Our results presented here showed that USP18 stimulated HBV production by inhibiting type I IFN signaling pathway independent of its protease activity. Although the precise mechanism and the involvement of host or viral molecules remain to be clarified, it raised the possibility of identifying USP18 as a potential therapeutic target in setting up a novel strategy of clinical protocols, especially for the treatment of $\mathrm{HBV}$-infected patients.

\section{Supplementary information}

Supplementary information accompanies this paper at https://doi.org/10. 1186/s12985-020-01304-2.

\section{Additional file 1: Supplemental Figure 1. Transfection efficiency} in HepAD38 cells. HepAD38 cells were seeded at $3 \times 10^{5} / \mathrm{ml}, 2 \mathrm{ml}$ per well in 6-well plates in antibiotic-free medium for $24 \mathrm{~h}$ before $1 \mu \mathrm{g}(\mathbf{A})$, $2 \mu \mathrm{g}(\mathbf{B}), 4 \mu \mathrm{g}(\mathbf{C})$ GFP plasmid DNA or $4 \mu \mathrm{g}$ empty vector (D) was transfected into each well. Fluorescent microscopy images were taken $48 \mathrm{~h}$ post transfection. Supplemental Figure 2. USP18 knockdown in HepAD38 cells did not affect expression of HBV proteins. HepAD38 cells were transfected with the $20 \mathrm{nM}$ siUSP18, $20 \mathrm{nM}$ negative siRNA or left untreated, respectively. Forty-eight hours later, culture medium was collected to quantify HBsAg (A) and HBeAg (B) expression level by ELISA assay. Intracellular HBcAg was detected by western blot $(\mathbf{C})$. Results are presented as means $\pm S D(n \geq 3)$. Supplemental Figure 3. IFN and ISG expression in HepAD38. HepAD38 cells were grown in the medium with or without tetracycline $(1 \mathrm{mg} / \mathrm{ml})$ until confluent for 6 days. IntracelIular RNA was then extracted. Expression of IFNa (A), IFNB (B) and ISGS mRNA including MxA (C) and OAS2 (D) were detected by real-time PCR. Results are presented as means $\pm \mathrm{SD}(\mathrm{n} \geq 3)$. ${ }^{*} p \leq 0.05 ;{ }^{* *} p \leq 0.01$; ${ }^{* *} p \leq$ 0.001 . Supplemental Figure 4. Detection of mycoplasma in HepAD38 cells. HepAD38 cells were grown in the medium with or without tetracycline $(1 \mathrm{mg} / \mathrm{ml})$ until confluent. The supernatant was collected for detecting mycoplasma. NC, negative control; PC, positive control; Tet+, the complete HepAD38 medium with $1 \mathrm{mg} / \mathrm{ml}$ tetracycline; Tet-, the complete HepAD38 medium without tetracycline.

\section{Abbreviations}

CHB: Chronic hepatitis B; GST: Glutathione S-transferase; HBV: Hepatitis B virus; HCC: Hepatocellular carcinoma; HCV: Hepatitis C virus; HNF: Hepatocyte nuclear factor; IFN: Interferon; IL-6: Interleukin 6; ISG15: Interferon stimulated gene 15; Jak/STAT: Janus kinase/signal transducer and activator of transcription; LCMV: Lymphocytic choriomeningitis virus; MxA: Myxovirus resistance-1; OAS2: 2',5'-oligo adenylate synthetase 2; PeglFN/Rib: Pegylated IFNa/ Ribavirin; PRRSV: Porcine respiratory and reproductive syndrome virus (PRRSV); USP18: Ubiquitin-specific protease 18; VSV: Vesicular stomatitis virus; WT-USP18: Wide type USP18

\section{Acknowledgements}

We thank Dr. Wenyu Lin (Massachusetts General Hospital,Harvard Medical School) for kindly providing ISRE-luc reporter plasmid and PRL-TK reporter plasmid.

\section{Authors' contributions \\ YJ and YM performed the main experiments and were major contributors in writing the manuscript. XD and $\mathrm{HY}$ analyzed the data statistically. SL constructed the USP18 plasmid used in the study. LC provide the primary idea of this study. YC participated in the design of this study and English editing of the revised manuscript. CY performed main experiments according to the reviewer's comments. All authors read and approved the final manuscript.}

\section{Funding}

This work is financially supported by grants from the CAMS Initiative for Innovative Medicine (CAMS-2016-I2M-3-025 and 2017-I2M-B\&R-15 to Limin Chen), Science and Technology Department of Sichuan Province, China (No.2017HH0024 to Limin Chen), Ministry of Science and Technology of China (2018YFE0107500 to Limin Chen) and Science and Technology 
Partnership Program, Ministry of Science and Technology of China(KY201904011 to Limin Chen).

\section{Availability of data and materials}

All data generated or analysed during this study are included in this published article.

\section{Ethics approval and consent to participate}

Not applicable.

\section{Consent for publication}

Not applicable.

\section{Competing interests}

The authors declare that they have no competing interests.

\section{Author details}

${ }^{1}$ Institute of Blood Transfusion, Chinese Academy of Medical Sciences and Peking Union Medical College, Chengdu 610052, Sichuan, China. ${ }^{2}$ The University of Hong Kong Shenzhen Hospital, Shenzhen 518053, China. ${ }^{3}$ Toronto General Research Institute, University Health Network, University of Toronto, Toronto, Ontario M5G1L6, Canada.

\section{Received: 24 June 2019 Accepted: 25 February 2020}

\section{Published online: 05 April 2020}

\section{References}

1. Liu YC, Penninger J, Karin M. Immunity by ubiquitylation: a reversible process of modification. Nat Rev Immunol. 2005;5:941-52.

2. Morales DJ, Lenschow DJ. The antiviral activities of ISG15. J Mol Biol. 2013; 425:4995-5008.

3. Dos Santos PF, Mansur DS. Beyond ISGlylation: functions of free intracellular and extracellular ISG15. J Interf Cytokine Res. 2017;37:246-53.

4. Malakhov MP, Malakhova OA, Kim Kl, Ritchie KJ, Zhang DE. UBP43 (USP18) specifically removes ISG15 from conjugated proteins. J Biol Chem. 2002;277: 9976-81.

5. Malakhova OA, Kim Kl, Luo JK, Zou W, Kumar KS, Fuchs SY, Shuai K, Zhang DE. UBP43 is a novel regulator of interferon signaling independent of its ISG15 isopeptidase activity. EMBO J. 2014;25:2358-67.

6. Chen L, Borozan I, Feld J, Sun J, Tannis L, Coltescu C, Heathcote J, Edwards A, McGilvray I. Hepatic gene expression discriminates responders and nonresponders in treatment of chronic hepatitis $C$ viral infection. Gastroenterology. 2005;128:1437-44.

7. Chen LM, Borozan I, Sun J, Guindi M, Fischer S, Feld J, Anand N, Heathcote $J$, Edwards AM, Mcgilvray ID. Cell-type specific gene expression signature in liver underlies response to interferon therapy in chronic hepatitis $C$ infection. Gastroenterology. 2010;138:1123-1133.e1123.

8. Xiao C, Qin B, Chen L, Liu H, Zhu Y, Lu X. Preactivation of the interferon signalling in liver is correlated with nonresponse to interferon alpha therapy in patients chronically infected with hepatitis B virus. J Viral Hepat. 2012;19:e1-e10.

9. Kim JH, Luo JK, Zhang DE. The level of hepatitis B virus replication is not affected by protein ISG15 modification but is reduced by inhibition of UBP43 (USP18) expression. J Immunol. 2008;181:6467.

10. Li L, Lei QS, Zhang SJ, Kong LN, Qin B. Suppression of USP18 potentiates the Anti-HBV activity of interferon alpha in HepG2.2.15 cells via JAK/STAT signaling. PLoS One. 2016;11:e0156496.

11. Ladner SK, Otto MJ, Barker CS, Zaifert K, Wang GH, Guo JT, Seeger C, King RW. Inducible expression of human hepatitis B virus (HBV) in stably transfected hepatoblastoma cells: a novel system for screening potential inhibitors of HBV replication. Antimicrob Agents Chemother. 1997;41:1715-20.

12. Chen L, Sun J, Meng L, Heathcote J, Edwards AM, McGilvray ID. ISG15, a ubiquitin-like interferon-stimulated gene, promotes hepatitis $C$ virus production in vitro: implications for chronic infection and response to treatment. J Gen Virol. 2009;91:382-8.

13. Quesada V, DíazPerales A, GutiérrezFernández A, Garabaya C, Cal S, LópezOtín C. Cloning and enzymatic analysis of 22 novel human ubiquitinspecific proteases. Biochem Biophys Res Commun. 2004;314:54-62.

14. Chen Z, Denison C, Huibregtse JM, Gygi S, Krug RM, Lambowitz AM. Human ISG15 conjugation targets both IFN-induced and constitutively expressed proteins functioning in diverse cellular pathways. Proc Natl Acad Sci U S A. 2005;102:10200-5
15. Zhu Y, Bo Q, Xi L, Chen L. Cell-type specific interferon stimulated gene staining in liver underlies response to interferon therapy in chronic HBV infected patients. Dig Dis Sci. 2012;57:2355-61.

16. Ritchie KJ, Hahn CS, Kim KI, Yan M, Rosario D, Li L, Jc DLT, Zhang DE. Role of ISG15 protease UBP43 (USP18) in innate immunity to viral infection. Nat Med. 2017:10:1374-8.

17. Nadine H, Namir S, Dong-Er Z, George I, Xu HC, Dieter HU, Mike R, Max LH, Lang PA, Lang KS. Usp18 driven enforced viral replication in dendritic cells contributes to break of immunological tolerance in autoimmune diabetes. PLoS Pathog. 2013;9(10):e1003650.

18. Ait-Ali T, Wilson AW, Finlayson H, Carré W, Westcott DG, Waterfall M, Frossard JP, Baek KH, Drew TW. Functional analysis of the porcine USP18 and its role during porcine arterivirus replication. Gene. 2009:439:35-42.

19. Xu D, Lillico SG, Barnett MW, Whitelaw CBA, Archibald AL, Ait-Ali T. USP18 restricts PRRSV growth through alteration of nuclear translocation of NF-KB p65 and p50 in MARC-145 cells. Virus Res. 2012;169:264-7.

20. Lok AS, McMahon BJ. Chronic hepatitis B: update 2009. Hepatology. 2009;50:661-2.

21. European Association for the Study of the Liver. EASL 2017 Clinical practice guidelines on the management of hepatitis B virus infection. J Hepatol. 2017(67):370-98.

22. Arimoto Kl, Lochte S, Stoner SA, Burkart C, Zhang Y, Miyauchi S, Wilmes S, Fan JB, Heinisch JJ, Li Z, et al. STAT2 is an essential adaptor in USP18mediated suppression of type I interferon signaling. Nat Struct Mol Biol. 2017;24:279-89.

23. Francois-Newton $V$, Magno de Freitas Almeida G, Payelle-Brogard B, Monneron D, Pichard-Garcia L, Piehler J, Pellegrini S, Uze G. USP18-based negative feedback control is induced by type I and type III interferons and specifically inactivates interferon alpha response. PLoS One. 2011;6:e22200.

24. Ran Y, Xuesen Z, Dawei C, Yuanjie L, Block TM, Ju-Tao G, Haitao G. The interferon-inducible protein Tetherin inhibits hepatitis $B$ virus Virion secretion. J Virol. 2015;89:9200.

25. Yan M, Jun Y, Chan HLY, Yang-Chao C, Hua W, Ying C, Chu-Yan C, Go MYY, Sau-Na T, Sai-Ming N. Glucose-regulated protein 78 is an intracellular antiviral factor against hepatitis B virus. Mol Cell Proteomics. 2009:8:2582-94.

26. Lu $X$, Wang J, Jin $X$, Huang $Y$, Zeng W, Zhu J. IFN-CSP inhibiting hepatitis B virus in HepG2.2.15 cells involves JAK-STAT signal pathway. Biomed Res Int. 2015;2015:959684.

27. Wieland S, Thimme R, Purcell RH, Chisari FV. Genomic analysis of the host response to hepatitis B virus infection. Proc Natl Acad Sci U S A. 2004;101: 6669-74.

28. Dunn C, Peppa D, Khanna P, Nebbia G, Jones M, Brendish N, Lascar RM, Brown D, Gilson RJ, Tedder RJ, et al. Temporal analysis of early immune responses in patients with acute hepatitis $B$ virus infection. Gastroenterology. 2009:137:1289-300.

29. Guidotti LG, Rochford R, Chung J, Shapiro M, Purcell R, Chisari FV. Viral clearance without destruction of infected cells during acute HBV infection. Science. 1999:284:825-9.

30. Gao D, Zhai A, Qian J, Li A, Li Y, Song W, Zhao H, Yu X, Wu J, Zhang Q. Down-regulation of suppressor of cytokine signaling 3 by miR-122 enhances interferon-mediated suppression of hepatitis B virus. Antivir Res. 2015;118:20-8.

31. Cheng X, Xia Y, Serti E, Block PD, Chung M, Chayama K, Rehermann B, Liang TJ. Hepatitis B virus evades innate immunity of hepatocytes but activates cytokine production by macrophages. Hepatology. 2017;66:1779-93.

32. Hosel M, Quasdorff M, Wiegmann K, Webb D, Zedler U, Broxtermann M, Tedjokusumo R, Esser K, Arzberger S, Kirschning CJ, et al. Not interferon, but interleukin-6 controls early gene expression in hepatitis B virus infection. Hepatology. 2009;50:1773-82.

33. Durfee LA, Huibregtse JM. Identification and validation of ISG15 target proteins. Subcell Biochem. 2010;54:228-37.

34. Villarroya-Beltri C, Guerra S, Sanchez-Madrid F. ISGylation - a key to lock the cell gates for preventing the spread of threats. J Cell Sci. 2017;130:2961-9.

35. Zhao C, Hsiang TY, Kuo RL, Krug RM. ISG15 conjugation system targets the viral NS1 protein in influenza a virus-infected cells. Proc Natl Acad Sci U S A. 2010;107:2253-8.

36. Hishiki T, Han Q, Arimoto K, Shimotohno K, Igarashi T, Vasudevan SG, Suzuki $Y$, Yamamoto N. Interferon-mediated ISG15 conjugation restricts dengue virus 2 replication. Biochem Biophys Res Commun. 2014:448:95-100.

37. Zhao $P$, Jiang $T$, Zhong $Z$, Zhao L, Yang $S$, Xia X. Inhibition of rabies virus replication by interferon-stimulated gene 15 and its activating enzyme UBA7. Infect Genet Evol. 2017;56:44-53. 
38. Zhao C, Sridharan H, Chen R, Baker DP, Wang S, Krug RM. Influenza B virus non-structural protein 1 counteracts ISG15 antiviral activity by sequestering ISGylated viral proteins. Nat Commun. 2016;7:12754.

39. Knobeloch KP, Utermohlen O, Kisser A, Prinz M, Horak I. Reexamination of the role of ubiquitin-like modifier ISG15 in the phenotype of UBP43deficient mice. Mol Cell Biol. 2005;25:11030-4.

40. Potu H, Sgorbissa A, Brancolini C. Identification of USP18 as an important regulator of the susceptibility to IFN-alpha and drug-induced apoptosis. Cancer Res. 2010;70:655-65.

\section{Publisher's Note}

Springer Nature remains neutral with regard to jurisdictional claims in published maps and institutional affiliations.

Ready to submit your research? Choose BMC and benefit from:

- fast, convenient online submission

- thorough peer review by experienced researchers in your field

- rapid publication on acceptance

- support for research data, including large and complex data types

- gold Open Access which fosters wider collaboration and increased citations

- maximum visibility for your research: over $100 \mathrm{M}$ website views per year

At BMC, research is always in progress.

Learn more biomedcentral.com/submissions 